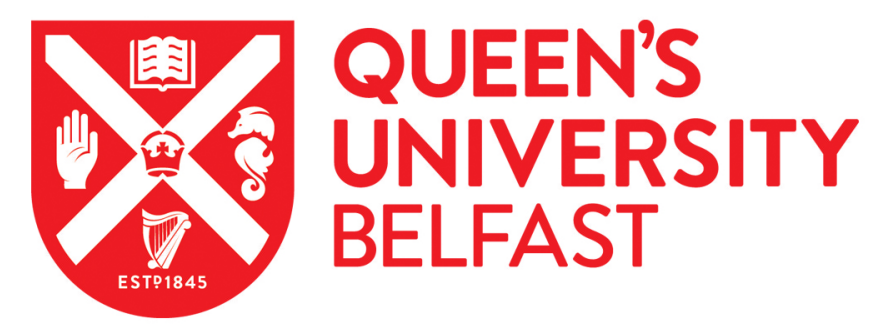

\title{
Compressive Sensing Multiplicative Antenna Array
}

Abbasi, M. A. B., Fusco, V., \& Zelenchuk, D. (2018). Compressive Sensing Multiplicative Antenna Array. IEEE Transactions on Antennas and Propagation, 66 (11), 5918-5925. [8432506].

https://doi.org/10.1109/TAP.2018.2864651

\section{Published in:}

IEEE Transactions on Antennas and Propagation

\section{Document Version:}

Peer reviewed version

Queen's University Belfast - Research Portal:

Link to publication record in Queen's University Belfast Research Portal

Publisher rights

(C) 2018 IEEE. This work is made available online in accordance with the publisher's policies. Please refer to any applicable terms of use of the publisher.

\section{General rights}

Copyright for the publications made accessible via the Queen's University Belfast Research Portal is retained by the author(s) and / or other copyright owners and it is a condition of accessing these publications that users recognise and abide by the legal requirements associated with these rights.

Take down policy

The Research Portal is Queen's institutional repository that provides access to Queen's research output. Every effort has been made to ensure that content in the Research Portal does not infringe any person's rights, or applicable UK laws. If you discover content in the Research Portal that you believe breaches copyright or violates any law, please contact openaccess@qub.ac.uk. 


\title{
Compressive Sensing Multiplicative Antenna Array
}

\author{
Muhammad Ali Babar Abbasi, Member IEEE, Vincent Fusco, Fellow, IEEE, Dmitry E. Zelenchuk, \\ Senior Member, IEEE
}

\begin{abstract}
This paper presents a novel approach to formulate an aggressively thinned sparse antenna array suitable for orthogonal multi-beam receiver applications. The power patterns of $M \times N$ element planar rectangular array are first reduced to orthogonally placed cross multiplicative sub-arrays. These arrays are then re-distributed using a compressive sensing (CS) approach in order to achieve array thinning along two 1-D sub-arrays for a fixed steered beam projection. A multi-beam synthesis approach is then implemented which permits efficient beam maxima as well as null placement of multiple interlaced far-field patterns. Numerical examples are presented to show the implementation of the proposed approach.
\end{abstract}

Index Terms - Multiplicative array, compressive sensing, beam space modulation, millimeter-wave, massive MIMO, 5 G

\section{INTRODUCTION}

$\mathrm{T}$ he increasing number of wireless devices required for future wireless communication system have multiplied data traffic volume. Various advanced wireless technologies have been developed and investigated over the last few years that have the potential to increase the capacity of a wireless communications system. Millimeter wave (mm-wave) technology is considered to be among the most notable candidate for the next generation communication systems because of promising features like high spectral efficiency [1], [2]. Other techniques such as beam space modulation [3]-[5] may also have a major role to play.

In general, as with all systems an increasing number of antenna elements means that number of associated RF chains required for beam forming also increases with attendant cost and power consumption penalties. One way to reduce the required expensive mm-wave equipment is by moving the basic signal processing close to the antennas. Some approaches (e.g. [6]-[8]) have investigated this and proposed the best choice of an array aperture.

The promising features of the sparse arrays (first reported about half a century ago [9]) have led to an intense research efforts in this field. Originally, synthesis strategies based on the iterative least squares [10], steepest-descent technique [11], and optimum autocorrelation function [12] were proposed. With the

Manuscript received XXX, revised XXX, published XXX (projected). This work was supported by the UK Engineering and Physical Science Research Council (EPSRC) under Grant EP/P000673/1 and EP/N020391/1.

M. Ali Babar Abbasi, Vincent Fusco and Dmitry Zelenchuk are with The Centre for Wireless Innovation (CWI), The Institute of Electronics, new developments in mm-wave arrays, a number of approaches based on finding a thinnest and sparsest configuration to match the desired pattern have started to surface. Thinned and sparse array synthesis is considered as a prolific field for evolutionary and stochastic optimization techniques, which require a definition of a suitable cost function. Some recent efforts aligned with this general idea used stochastic approximation [13], nested optimization [14] and iterative techniques [15]. Recent research efforts have shown to outperform previous approaches in terms of required radiation characteristics and convergence speed. Some of the most notable techniques include genetic algorithm (GA) [16], [17], iterative Fourier transform (IFT) [10], [18], particle swarm (PS) [19]-[21], multi-level branch-and-bound (B\&B) [22] matrix pencil method [23], Compressive sensing [24], [25], invasive weed (IW) [26], ant colony [27] and so on. A number of hybrid schemes of multiple approaches were also proposed [28], [29]. It is noteworthy that although these approaches show effectiveness in reproducing the desired/reference patterns, some do not work for asymmetrical beams. Some recent advance methods like Bayesian compressive sensing has overcome this problem too [30]. Achieving a steerable thinned and sparse array solution requires a fully connected array hardware with RF switches where the beam projection is controlled by phase shifters or periodic time sequencing. The primary goal for this paper is to develop an aggressive thinned antenna array synthesis approach that preserves the ability to create a multiplicity of orthogonal beams located at any position in $u, v$ space. To facilitate this we first map a 2-dimensional (2D) array to a multiplicative array, [31] and then show how the number of array elements from which it is comprised can be further reduced by using compressive sensing. Next we propose a multi-beam orthogonal beam space synthesis method, based on a directional modulation approach, [32], [33], and demonstrate an example three beam solution. In contrast to dynamically steerable beam satisfying a priori reference field pattern [22], [26], [34], [35] the proposed approach focus only on the fixed beam operation which requires minimum hardware cost. Theoretical formulation of the approach is presented in section II, implementation of the approach with an aid of a

Communications and Information Technology (ECIT), School of Electronics, Electrical Engineering and Computer Science (EEECS), Queen's University Belfast, Belfast BT3 9DT, United Kingdom (email: m.abbasi@qub.ac.uk, v.fusco@ecit.qub.ac.uk, d.zelenchuk@qub.ac.uk) 


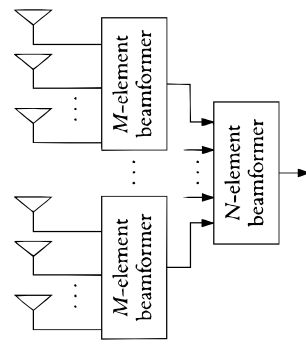

(a)

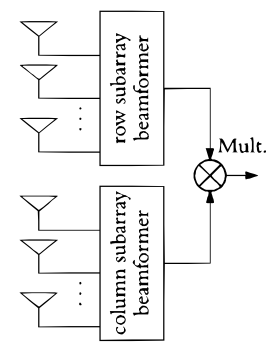

(b)
Fig. 1. Architecture of (a) conventional uniform planar rectangular array and (b) multiplicative array.

numerical example and corresponding results are discussed in section III, while the findings are concluded in section IV of the paper.

\section{ARRAY THINNING ANALYSIS}

\section{A. Multiplicative Array Synthesis}

Consider a planar $M \times N$ element 2-D uniformly distributed array. The array factor consisting of isotropic antenna elements in the $x y$-plane can be defined as

$A F(\theta, \phi)=\sum_{m=0}^{M-1} I_{m x} e^{j\left(k m d_{x} \sin \theta \cos \phi+a_{m x}\right)} \sum_{n=0}^{N-1} I_{n y} e^{j\left(k n d_{y} \sin \theta \sin \phi+a_{n y}\right)}$

where $I_{m x}$ and $I_{n y}$ are the complex excitation amplitude of antenna elements, $a_{m x}$ and $a_{n y}$ represents the phase shifts between antenna elements, $d_{x}$ and $d_{y}$ are the periods with respect to $x$ and $y$ axes. Under monochromatic excitation, element spacing must be no greater than a half-wavelength in order to prevent unwanted grating lobes so an additional condition $d_{x}, d_{y}$ $\leq \lambda / 2$ applies. The terms $\sin \theta \cos \phi$ and $\sin \theta \sin \phi$ are replaceable by $u$ and $v$ respectively. The array factor in the $+z$ hemisphere can then be written as [36]

$$
\mathbf{F}(u, v)=\mathcal{F}^{-1}\left(\mathbf{w}_{m x}{ }^{t} \cdot \mathbf{w}_{n y}\right)
$$

where $(\cdot)$ denotes the vector multiplication, $\mathcal{F}^{-1}$ is the inverse Fourier transform, superscript $t$ denotes the transpose, $\mathbf{w}_{m x}$ and $\mathbf{w}_{\text {ny }}$ are the complex weight vectors of the 2-D rectangular array, when $m=1,2,3 \ldots M$, and $n=1,2,3 \ldots N$, distributed along the $x$ and $y$ directions, respectively. Equation (2) can be rewritten as

$$
\mathbf{F}(u, v)=\mathcal{F}^{-1}\left(\mathbf{w}_{m x}{ }^{t}\right) \cdot \mathcal{F}^{-1}\left(\mathbf{w}_{n y}\right)
$$

and hence the power patterns of a uniform rectangular array as

$$
\mathbf{P}=\mathbf{F}(u, v) \times \mathbf{F}(u, v)^{*}
$$

when $(\times)$ represents element by element (or Hadamard [37]) multiplication while superscript $(*)$ is the complex conjugate operation. Using the distributive property of the Fourier Transform, we rewrite equation (4) as

$$
\mathbf{P}=\left(\mathcal{F}^{-1}\left(\mathbf{w}_{m x}{ }^{t}\right) \times\left[\mathcal{F}^{-1}\left(\mathbf{w}_{m x}{ }^{t}\right)\right]^{*}\right) \cdot\left(\mathcal{F}^{-1}\left(\mathbf{w}_{n y}\right) \times\left[\mathcal{F}^{-1}\left(\mathbf{w}_{n y}\right)\right]^{*}\right)
$$

The above equation reveals that by replacing the multiplication with an auto-convolution of the complex weighting elements, we can reproduce the power patterns of a planar rectangular array by only using two orthogonal 1-D arrays [38]. The expression can be written as

$$
\mathbf{P}=\left(\mathcal{F}^{-1}\left(\mathbf{w}_{m x}{ }^{t} \otimes \mathbf{w}_{m x}{ }^{t}\right)^{*}\right) \cdot\left(\mathcal{F}^{-1}\left(\mathbf{w}_{n y} \otimes \mathbf{w}_{n y}\right)\right)
$$

when $\otimes$ represents the one dimensional convolution operation. To further elaborate, the power patterns of $M \times N$ uniformly distributed arrays can be reproduced by auto convolution of complex weight vectors when

$$
\begin{aligned}
\boldsymbol{w}_{x}^{t} & =\mathbf{w}_{m x} \otimes \mathbf{w}_{m x}=\left[\begin{array}{lllll}
w_{x 1} & w_{x 2} & w_{x 3} & \cdots & w_{x(2 M-1)}
\end{array}\right]^{t} \\
\boldsymbol{w}_{y} & =\mathbf{w}_{n y} \otimes \mathbf{w}_{n y}=\left[\begin{array}{lllll}
w_{y 1} & w_{y 2} & w_{y 3} & \cdots & w_{y(2 N-1)}
\end{array}\right]
\end{aligned}
$$

This approach can be used to decrease the number of 2-D array elements from $M \times N$ to two single $(2 M-1)+(2 N-1)$ element orthogonal arrays as suggested by $1-\mathrm{D}$ convolution. In order for the desired spatial power pattern function to be realized, a further post processing unit at the receiver is required for the combination of the two 1-D element patterns. Note that there are two conditions for equation (6) to hold: 1) the physical placement of the two 1-D sub-array elements should be orthogonal to each other and 2) the element weight magnitude should be distributed symmetrically around the 1-D sub-array phase center as expressed through

$$
\mathcal{F}^{-1}\left(\mathbf{w}_{x, y}\right)=\left[\mathcal{F}^{-1}\left(\mathbf{w}_{x, y}\right)\right]^{*}
$$

The above mentioned procedure suggests that the power patterns obtained from the approach in Fig. 1(b) should yield the same power patterns using significantly lower number of RF chains than required for the full 2-D array (Fig. 1(a)). For arrays with a small number of antenna elements e.g. $M=N=4$ the number of elements required by the scheme will be 14, while the corresponding planar rectangular array will have 16 elements. This situation changes dramatically for a higher number of array elements, e.g. for $N=M=20$, the rectangular array will have 400 elements and the corresponding multiplicative array will require only 78 elements.

\section{B. Compressive Sensing Implementation}

In this section we will show that the number of elements in each of the two 1-D arrays of the multiplicative cross formation and hence associated RF chains can be further reduced. It has been shown in Section A that the synthesized pattern is governed by the complex weights $\boldsymbol{w}_{x}$ and $\boldsymbol{w}_{y}$, and the distances between two antenna elements $\left(m d_{x}\right.$ and $\left.n d_{y}\right)$ representing the relative position of each antenna element from phase center in $x-y$ plane. The actual power patterns of symmetrically distributed uniform array elements is as given by (5). Consider now what happens when the distributed antenna elements in a given $x y$-plane space are mapped to a $\mu \times v$ space where:

$$
\begin{gathered}
\mu=1,2,3 \cdots M^{\prime} \\
\nu=1,2,3 \cdots N^{\prime}
\end{gathered}
$$

When $M^{\prime}=4 M^{2}$ and $N^{\prime}=4 N^{2}$. We divided $\lambda / 2$ into $2 M-1$ and $2 N-1$ segments such that the element spacing in $\mu \times v$ space is given by $\delta_{x}=d_{x} /(2 M-1)$ and $\delta_{y}=d_{y} /(2 N-1)$. In other words, we distribute the antenna elements in such a way that two consecutive elements are collocated at a distance $\delta$ when every radiating elements is located at $d_{x}$ and $d_{y}$, while non-radiating elements are located elsewhere in $\mu \times v$ space. This space can 


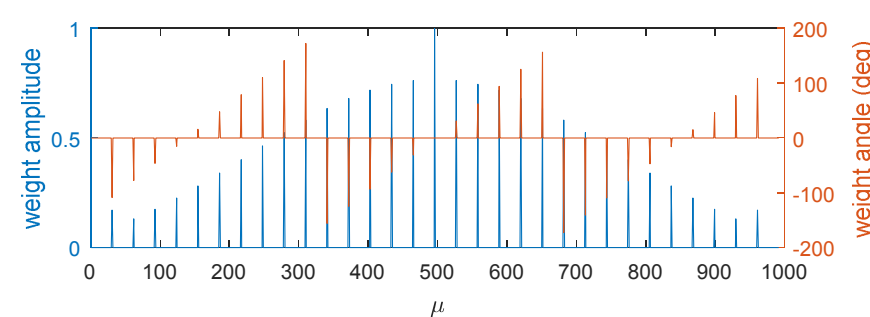

(a)

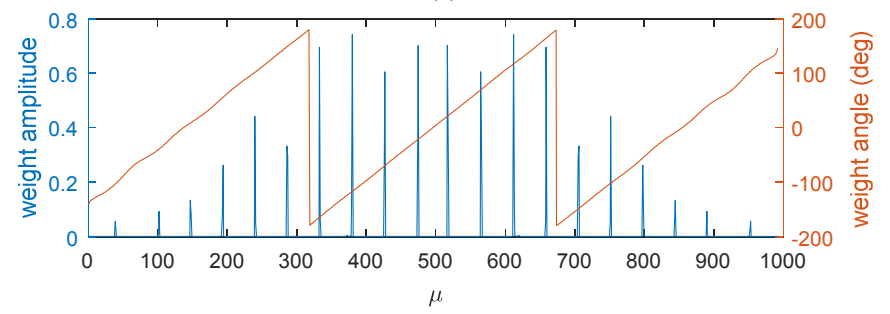

(b)
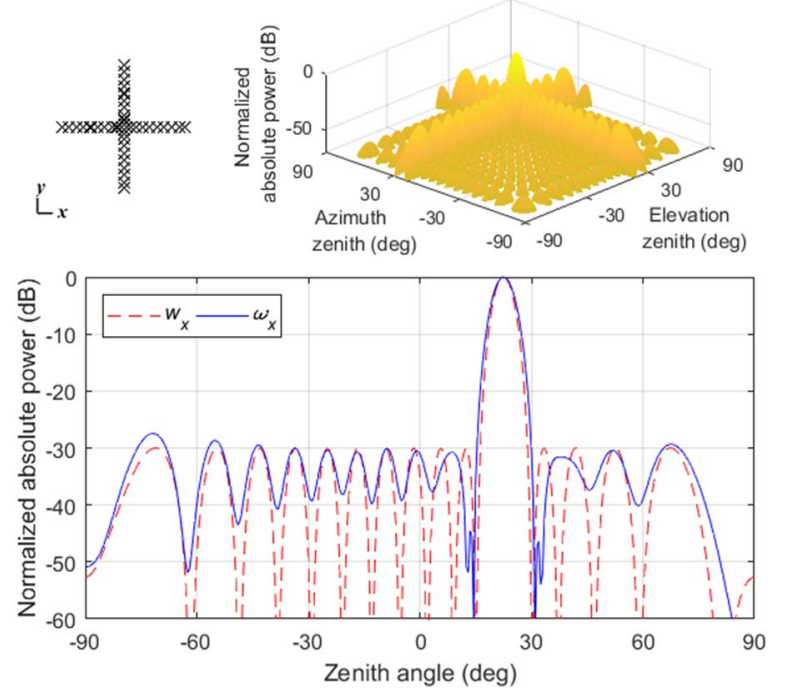

(c)

Fig. 2. (a) $\boldsymbol{w}_{x}$ magnitude and phase of equally spaced antenna elements for the intended beam direction $\theta=22.5^{\circ}$, (b) $\omega_{x}$ magnitude and phase of unequally spaced sparse elements distributed along vector $\mu$, (c) Top left: array architecture, top right: 2-D rectangular resultant power pattern of the array, and bottom: comparison of 1-D cuts of the resultant 2-D power patterns.

now be exploited as an a priori requirement for the compressive sensing approach in which the algorithm can compress the number of antenna elements and re-distribute them in $\mu \times v$ space. The 2-D power pattern cut in this case can be represented along the $\theta$ plane by

$$
[\mathbf{P}(\theta)]_{1 \times S x}^{t}=\left[\boldsymbol{\omega}_{x}\right]_{1 \times M^{\prime}}^{t} \times\left[\mathbf{H}_{x}\right]_{M^{\prime} \times S x}^{t}
$$

and along the $\phi$ plane by

$$
[\mathbf{P}(\phi)]_{1 \times S y}=\left[\boldsymbol{\omega}_{y}\right]_{1 \times N^{\prime}} \times\left[\mathbf{H}_{y}\right]_{N^{\prime} \times S y}
$$

where $P(\theta)$ and $P(\phi)$ are the power pattern cuts, $\boldsymbol{\omega}_{x}$ and $\boldsymbol{\omega}_{y}$ are the complex excitation vector of the antenna elements, $s x=$ $1,2,3 \ldots S x$ and $s y=1,2,3 \ldots S y$ represents the number of data samples of the pattern along $x$ and $y$ axis respectively, $\mathbf{H}$ is a matrix consisting of the steering vectors.

Minimization of vector $\omega$ can be considered as a standard basis pursuit problem, which aims to find a sparse solution.
Typically, although such a problem is ill-posed [39] it can be formulated as a convex optimization problem (also known as base pursuit (BP)) of the form

$$
\min \|\omega\|_{1} \text { subject to } \mathbf{P}=\boldsymbol{\omega} \mathbf{H}
$$

This synthesis problem can be considered in 2 ways:

1- Minimize the side lobe level subject to minimum number of array elements

2- Minimize the maximum side lobe level subject to fixed number of array elements

In first case, typically, it is desired to match the given patterns to a given level of accuracy, so the algorithm needs to generate a minimum number of elements with corresponding excitation matrix for which the resultant pattern should confine within a given mask of reference patterns. An approach called convex relaxation $(\mathrm{CR})$ that is based on $\mathrm{BP}$ can be alternatively used to re-formulate the problem as a basis pursuit denoise (BPDN) problem [40]

$$
\min \|\omega\|_{1} \text { subject to }\|\mathbf{P}-\omega \mathbf{H}\|_{l 2} \leq \xi
$$

where vector $\boldsymbol{\omega}$ is considered as the optimal solution of the problem (15) if it has the smallest objective value among all vectors that satisfy the given constraints. The positive parameter $\xi$ is the pre-defined relaxation factor or a predictionobservation discrepancy. For simplicity the Euclidean norm is considered in this study to define the CS convergence criteria. The success of such a formulation is motivated by the fact that the unique solution for a specific case where $\xi=0$ exactly coincides to the BP i.e., a basis pursuit solution given in (14). Moreover, the convexity of this formulation enables the use of computationally tractable algorithms to find efficient solutions. Based on the same framework, very powerful and well matured packages have been developed in recent years (the well-known L1-Magic tool is one of the many examples [41]). The formulation (15) is used to find a sparse solution which enables the vectors $\omega_{x}$ and $\omega_{y}$ to update in every iteration.

An example is presented in Fig. 2. Here we define a 31 element $\boldsymbol{w}_{x}-$ Dolph-Chebyshev excitation vector with $d_{x}=0.5 \lambda$, sidelobe level (SLL) $=-30 \mathrm{~dB}$ and main beam direction at $\theta=$ $22.5^{\circ}$. The vector $\boldsymbol{w}_{x}$ was distributed along vector space $\mu$ as shown in Fig. 2(a). For brevity, only the array weights corresponding to the sub-array along the $x$-axis are shown. Fig. 2(b) shows the compressive sensing algorithm implementation where 31 antenna elements are shown to be reduced to 20 elements (64.5\% thinning), distributed along $\mu$ space, at $\xi=10$

- The number of algorithm iterations were 23. All the computations were carried out on intel-i7 $3.4 \mathrm{GHz}$ with $32 \mathrm{~GB}$ RAM with SSD. Comparing $\boldsymbol{w}_{x}$ and $\boldsymbol{\omega}_{x}$ reveals that the magnitude as well as the phase information is preserved everywhere along $\mu$, however, when the $\boldsymbol{\omega}_{x}$ magnitude $\rightarrow 0$, the phase information can be neglected.

The resultant normalized power patterns of an array excited by $\boldsymbol{w}$ and $\boldsymbol{\omega}$ distributed in multiplicative cross formation is presented in Fig. 2(c) (top left). We consider array distribution along $x y$-plane radiating in the forward half space $+z$. The azimuth and elevation planes are defined to be along $x z$ - and $y z$ planes respectively. For ease in understanding, we consider zenith angle $=0^{\circ}$ representing the broadside direction, where we define $\phi$ and $\theta=0^{\circ}$. The sampling distance along the $\theta$ and $\phi$ region was set to $0.5^{\circ}$ and for ease of comparison a 1-D cut of 


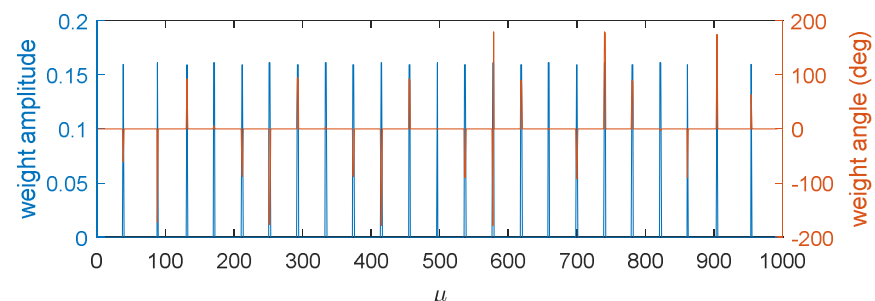

(a)

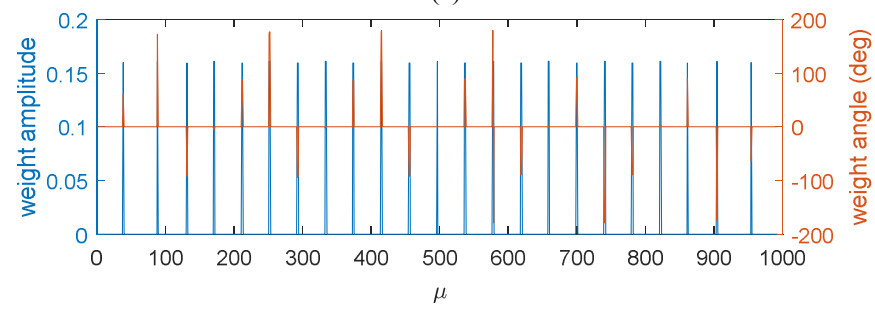

(b)

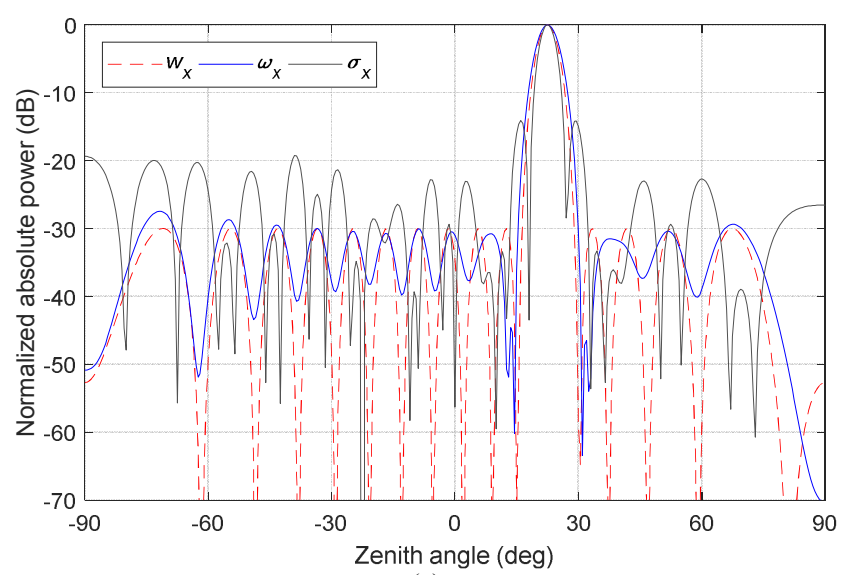

(c)

Fig. 3. $x$-axis represents the vector $\mu$ and $y$-axis represents the calculated magnitude and phase of (a) $\sigma_{\alpha x}$ and (b) $\sigma_{\beta x}$ and (c) 1-D cuts of resultant 2-D power patterns.

the 2-D pattern (Fig. 2(c) top right) is shown. The agreement between the resultant patterns for $\boldsymbol{w}$ and $\boldsymbol{\omega}$ which is governed by the system requirements depends upon the choice of $\xi$. As mentioned before, the optimal solution $\omega$ yields an updated spacing between the elements. However, since physical antenna elements occupy actual space, this solution sometimes prevent practical realization as some element positions can be very close together. The inclusion of spacing constraints into the formulation in (15) can further improve the solution in order to accommodate the dimensions of physical radiating elements. This is incorporated by post processing the solution $\omega$. For example, for a given solution, if $d_{m}-d_{m+1} \ll d_{m}$, we approximate two elements with a single element excited by the vector summation of the two complex excitation weights of the parent elements.

\section{Steered Direction Multiplicative Array}

Looking closely at the multiplicative array topology in Fig. 2(c) (top left), it is evident that only a small portion of physical aperture is utilized as compared to a fully-filled rectangular array. Also the 1-D sub-arrays along $\mu$ and $v$ respectively do not necessarily need to coincide at their phase centers provided they are kept orthogonal to each other. These two facts permit

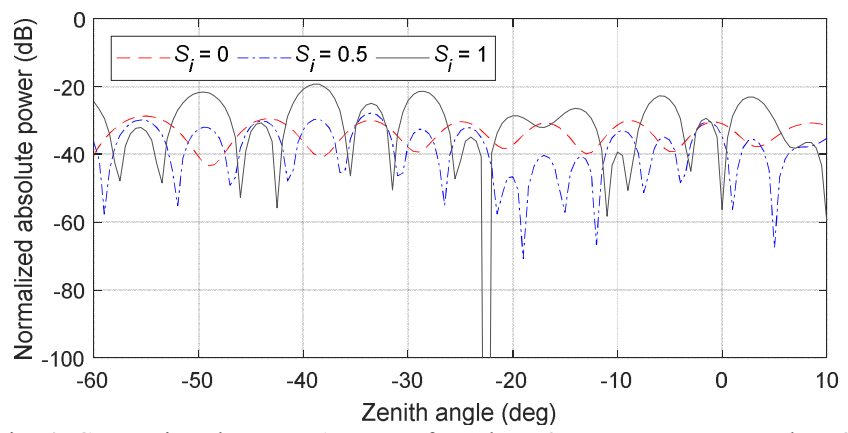

Fig. 4. Comparison between 1-D cut of resultant 2-D power patterns when $S_{i}$ is changed from 0 to 1 .

considerable flexibility in the choice of array topology. In the light of the above, we propose an orthogonal beam synthesis strategy using the element locations governed by $\omega_{x}$ and $\omega_{y}$. We further propose to host multiple nested multiplicative arrays and thus permit acquisition of multi-beam signals.

To formulate this, we consider an example array aperture hosting two sparse and thinned multiplicative cross arrays. The array radiating in a particular direction $(\alpha)$ needs to provide a null that coincides with the far field power maxima of second array (in $\beta$ direction) when $\alpha \neq \beta$. Both arrays are hosted within the same array aperture. The approach used in [32] is adapted so that the excitation vector based on the pattern projection method is given by $\sigma_{\alpha}$ and $\sigma_{\beta},(16),(17)$. For simplicity the pattern projection along in pre-specified directions and is given as

$$
\begin{aligned}
& \boldsymbol{\sigma}_{\alpha x}=\left[\begin{array}{lllll}
\sigma_{\alpha 1} & \sigma_{\alpha 2} & \sigma_{\alpha 3} & \cdots & \sigma_{\alpha M^{\prime}}
\end{array}\right]^{t} \\
& =\frac{1}{\left\|\mathbf{D}_{\theta}(\alpha)\right\|^{2}}\left[\mathbf{I}_{M^{\prime}}-\left(\mathbf{D}_{\theta}^{\dagger}(\beta)\right)^{-1} \mathbf{D}_{\theta}^{\dagger}(\beta)\right] \mathbf{D}_{\theta}(\alpha) \\
& \boldsymbol{\sigma}_{\beta x}=\left[\begin{array}{lllll}
\sigma_{\beta 1} & \sigma_{\beta 2} & \sigma_{\beta 3} & \cdots & \sigma_{\beta M^{\prime}}
\end{array}\right]^{t} \\
& =\frac{1}{\left\|\mathbf{D}_{\theta}(\beta)\right\|^{2}}\left[\mathbf{I}_{M^{\prime}}-\left(\mathbf{D}_{\theta}^{\dagger}(\alpha)\right)^{-1} \mathbf{D}_{\theta}^{\dagger}(\alpha)\right] \mathbf{D}_{\theta}(\beta)
\end{aligned}
$$

Where the operation superscript $(-1)$ represents the MoorePenrose Pseudoinverse, $\mathbf{I}_{M^{\prime}}$ denotes the $M^{\prime} \times M^{\prime}$ identity matrix, and the superscript ' $\dagger$ ' denotes the complex conjugate transpose (Hermitian) operator. $\mathbf{D}_{\theta}(\alpha)$ and $\mathbf{D}_{\theta}(\beta)$ are the vectors [33] along the directions $\alpha$ and $\beta$, and can be written as

$$
\begin{aligned}
& \mathbf{D}_{\theta}(\alpha)=\left[\begin{array}{llll}
e^{j \frac{M^{\prime}-1}{2} \pi \cos \alpha} & e^{j\left(\frac{M^{\prime}-1}{2}-1\right) \pi \cos \alpha} & \cdots & e^{j\left(\frac{M^{\prime}-1}{2}-\left(M^{\prime}-1\right) \pi \cos \alpha\right.}
\end{array}\right]^{t} \\
& \mathbf{D}_{\theta}(\beta)=\left[\begin{array}{llll}
e^{j \frac{M^{\prime}-1}{2} \pi \cos \beta} & e^{j\left(\frac{M^{\prime}-1}{2}-1\right) \pi \cos \beta} & \cdots & e^{j\left(\frac{M^{\prime}-1}{2}-\left(M^{\prime}-1\right) \pi \cos \beta\right.}
\end{array}\right]^{t}
\end{aligned}
$$

It is important to highlight that the pattern projection vectors $\sigma_{\alpha}$ and $\sigma_{\beta}$ need to be distributed in $\mu \times v$ space, and to coincide with $\boldsymbol{\omega}_{x}$ and $\boldsymbol{\omega}_{y}$ for realizable physical placement of practical physical sized antenna elements. In continuation to the example presented in Fig. 2, Fig. 3(a) shows the calculated $\sigma_{\alpha x}$ for main beam direction selected to lie at $\theta=22.5^{\circ}$ and a null direction located at $\theta=-22.5^{\circ}$, while Fig. 3(b) shows $\sigma_{\beta x}$ for main beam direction positioned at $\theta=-22.5^{\circ}$ and a null direction along $\theta$ $=22.5^{\circ}$. Again, only the sub-array distributed along $\mu$ is shown . 


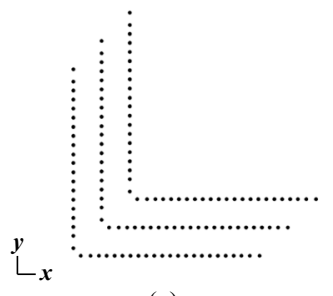

(a)

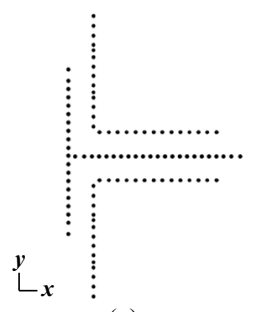

(c)

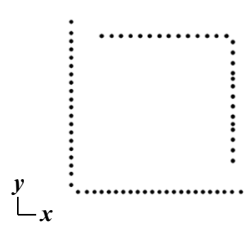

(b)

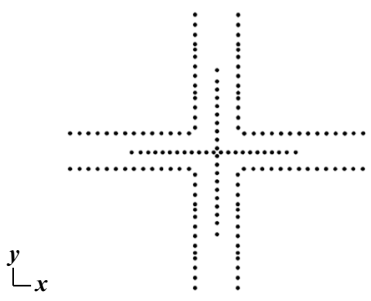

(d)
Fig. 5. Possible architectures of the proposed multi-beam multiplicative antenna array where each element's excitation is defined by $\mathbf{E}_{i}$ for the $i$ th array when (a) $i=3$, (b) $i=2$, (c) $i=3$, and (d) $i=5$.

The excitation vectors $\sigma_{\alpha}$ and $\sigma_{\beta}$ coincide with $\omega$ where $|\omega|>$ 0 . The resultant normalized power patterns for $\sigma_{\alpha}$ are compared in Fig. 3(c) where the main beam and the side lobes are shown. The power distribution set by $\boldsymbol{w}_{x}$ where SLL is confined below $-30 \mathrm{~dB}$ is tracked by $\omega_{x}$ to an acceptable degree. However, for the case $\sigma_{\beta x}$ this power distribution resulted in the maximum SLL moving from $-27.5 \mathrm{~dB}$ (for the case of $\omega_{x}$ ) to $19.3 \mathrm{~dB}$ at an expense of a perfect null, along $\theta=-22.5$. Considering this effect, the final excitation matrix $\mathbf{E}_{i}$ for a given array can be defined as

$$
\mathbf{E}_{i}=\omega-S_{i} \omega+S_{i} \boldsymbol{\sigma}
$$

where $S_{i}\left(0 \leq S_{i} \leq 1\right)$ is a scaling factor which decides the contribution of null placements defined by $\sigma$ for the $i$ th array. The choice of $S_{i}$ defines the compromise between maximum allowable SLL and accurate null depth as shown in Fig. 4 where the side lobes with almost fairly-distributed power (at $S_{i}=0$ ) are transformed to an un-equal power levels with a perfect null at $-22.5^{\circ}$ at $S_{i}=1$.

\section{2-D Array Lattice Choices}

Assume an array aperture hosting $i$ nested arrays, each projecting orthogonal beams in multiple directions (say along $\alpha, \beta, \gamma \ldots)$. Based on the approach described above many different topologies of arrays in such an aperture are possible. Consider Fig. 5(a), here three 2-D quasi-(L) arrays are nested. This architecture is suitable when the coupling impact between orthogonal arrays is to be minimal. In (Fig. 5(b)), only the corner antenna elements of $i$ arrays are close to each other, adding benefits of low mutual coupling. More complex array architectures like the ones shown in Fig. 5(c) and (d) are also feasible. In all cases the number of compressed antenna array elements depends upon the required half power beam width (HPBW) in a given direction (e.g. $\alpha$ ) which defines the required physical size of an array. When the HPBW for each array can be different a possible approach is to first create the quasi-(L) or quasi- $(+)$ formation for an array requiring the narrowest HPBW, then to use the available space on the aperture to host a second array which requires the second narrowest HPBW (e.g. another quasi-(L) architecture, Fig.5(a)) and so on.

\section{NUMERICAL EXAMPLE AND RESULTS}

In section II, we discussed the theoretical formation of the proposed approach with the help of examples. In this section we show a sample orthogonal multi-beam synthesis. For this we have taken the array lattice as shown in Fig.5(a) in which three arrays are defined $(i=3)$. We assigned a non-symmetric and distinct beam directions $\alpha=\left[-22.5^{\circ},-40^{\circ}\right], \beta=\left[35^{\circ}, 40^{\circ}\right]$ and $\gamma$ $=\left[10^{\circ},-10^{\circ}\right]$ to each array. First, Dolph-Chebyshev excitation vector with $d_{x}=0.5 \lambda, \mathrm{SLL}=-30 \mathrm{~dB}$ was used to create 16 element $\boldsymbol{w}_{x}$ and $\boldsymbol{w}_{y}$. The compressive sensing algorithm with $\xi=5$ was then deployed to define $\omega_{x}$ and $\omega_{y}$. Finally, beam space modulation was implemented to define $\mathbf{E}_{1}, \mathbf{E}_{2}$ and $\mathbf{E}_{3}$ for each array. $S_{i}$ was chosen to be 0.9 to avoid un-realistic deep nulls [42]. The final array isotropic element populated architecture is shown in Fig. 6(a) where the outer most 26 element array is named "array 1", 28 elements central array is "array 2", and 24 element inner most array is "array 3". Each antenna element location and associated complex excitation weights are tabulated in Table I. Note that where multiple closely spaced antenna elements occur, as governed by $\omega$ for each array, these were replaced by a single antenna element. After combination as in Fig.1(b), the rectangular normalized power patterns of all three arrays were evaluated and are presented in Fig. 6(b)-(d). In Fig. 6(b), the main beam projection is evident along the direction $\alpha$ while, two nulls are located along the directions $\beta$ and $\gamma$ represented as vertical contours. Similarly, power patterns for main beam along $\beta$ and $\gamma$ are shown in Fig. 6 (c) and (d). 1-D cuts of the 2-D plots are presented in Fig. 6(e) and (f) for ease in comparison. Each array projects a beam in a pre-defined direction, while simultaneously providing nulls along the main beam projection directions of the other two remaining arrays. Presence of the multiplicative block in array architecture shown in Fig. 1(b) is primarily responsible for the side lobe level along the principle planes in all the power patterns. The final response validates the theoretical predictions formulated in Section II with acceptable minor deviations in null placement (last column in Table I). This deviation is primarily because of the simple approximation method used to unite very closely spaced elements. The same approach is scalable to any number of antenna elements sharing the same physical aperture with multiple beam projections. The main array metrics and computational effort required are presented in Table II.

In general, sparse distribution of antenna elements in a 2-D planar array lattice is carried out in an attempt to match 2-D reference power patterns (e.g. [26], [34], [22]). In contrast to these approaches, in this paper we use a multiplicative array lattice with multi-beam directional modulation implementation (Fig. 5), which makes the proposed method one of a kind. Moreover, the multiple interlaced orthogonal power patterns with strategically placed nulls (Fig. 6(b) - (d)) limits the comparability of the proposed lattices (Fig. 5) with other 


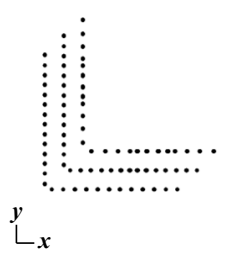

(a)

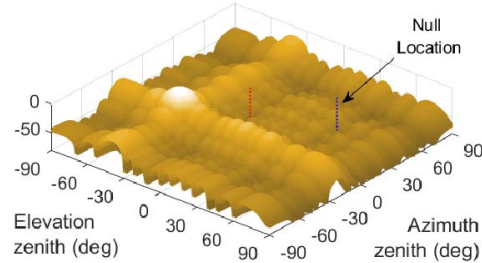

(b)

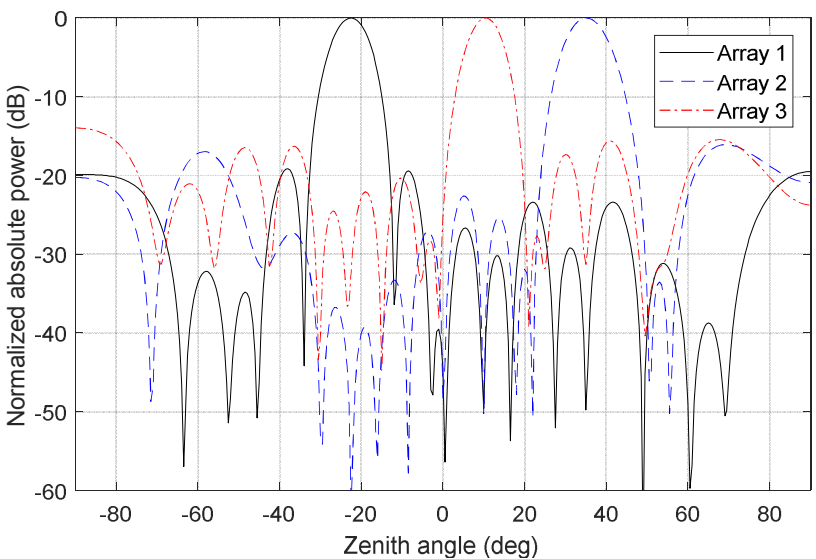

(e)

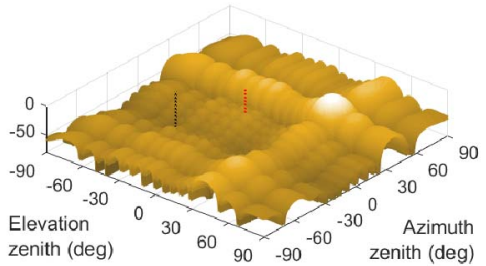

(c)

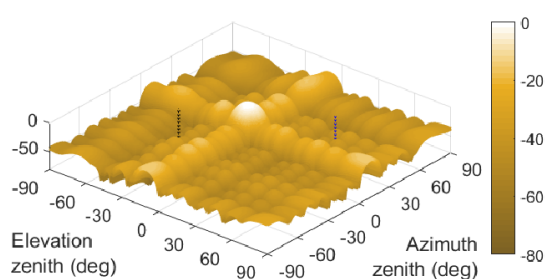

(d)

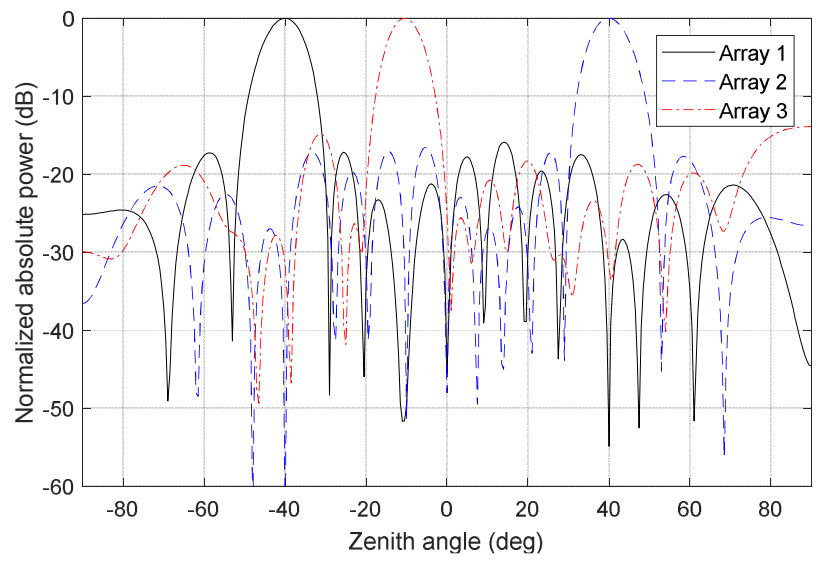

(f)

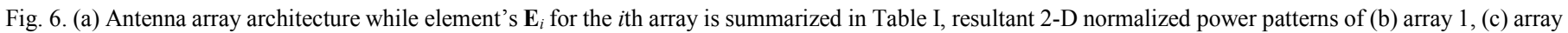

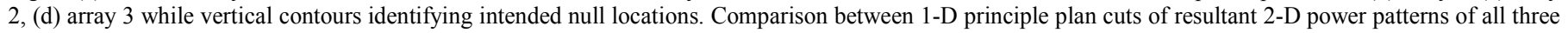
arrays (e) along azimuth, and (f) along elevation.

TABLE I

Complex Excitation Weights $\mathbf{E}_{1}, \mathbf{E}_{2}, \mathbf{E}_{3}$ and Antenna Element Physical Placements

\begin{tabular}{|c|c|c|c|c|c|c|c|c|c|c|c|c|}
\hline \multirow{3}{*}{$\begin{array}{c}\text { Element } \\
\text { No. }\end{array}$} & \multicolumn{4}{|c|}{ Position $(\lambda)$} & \multirow{2}{*}{\multicolumn{2}{|c|}{ Array 3}} & \multicolumn{3}{|c|}{ Magnitude } & \multicolumn{3}{|c|}{ Phase (rad) } \\
\hline & \multicolumn{2}{|c|}{ Array 1} & \multicolumn{2}{|c|}{ Array 2} & & & Arrav 1 & Array 2 & Array 3 & Array 1 & Arrav 2 & Arrav \\
\hline & $x$ & $y$ & $x$ & $y$ & $x$ & $y$ & Array 1 & Array 2 & Array 3 & Array 1 & Array 2 & \\
\hline 1 & 0.37 & 0 & 1.33 & 1 & 2.45 & 2 & 0.197 & 0.189 & 0.388 & -1.72 & -0.407 & -2.711 \\
\hline 2 & 0.97 & 0 & 1.88 & 1 & 3.18 & 2 & 0.25 & 0.31 & 0.362 & -0.212 & -2.348 & 2.689 \\
\hline 3 & 1.58 & 0 & 2.42 & 1 & 3.85 & 2 & 0.402 & 0.337 & 0.45 & 1.2 & 1.756 & 2.059 \\
\hline 4 & 2.18 & 0 & 2.98 & 1 & 4.48 & 2 & 0.49 & 0.4 & 0.395 & 2.695 & -0.236 & 1.437 \\
\hline 5 & 2.78 & 0 & 3.48 & 1 & 4.87 & 2 & 0.496 & 0.445 & 0.491 & -2.12 & -1.964 & 0.88 \\
\hline 6 & 3.38 & 0 & 4 & 1 & 5.37 & 2 & 0.491 & 0.56 & 0.597 & -0.826 & 2.586 & 0.268 \\
\hline 7 & 3.95 & 0 & 4.47 & 1 & 5.97 & 2 & 0.492 & 0.513 & 0.596 & 0.762 & 0.795 & -0.238 \\
\hline 8 & 4.55 & 0 & 4.87 & 1 & 6.47 & 2 & 0.496 & 0.513 & 0.491 & 2.053 & -0.692 & -0.847 \\
\hline 9 & 5.15 & 0 & 5.33 & 1 & 6.85 & 2 & 0.49 & 0.561 & 0.395 & -2.764 & -2.481 & -1.407 \\
\hline 10 & 5.75 & 0 & 5.85 & 1 & 7.48 & 2 & 0.402 & 0.445 & 0.45 & -1.271 & 2.066 & -2.028 \\
\hline 11 & 6.37 & 0 & 6.35 & 1 & 8.15 & 2 & 0.25 & 0.399 & 0.362 & 0.14 & 0.34 & -2.656 \\
\hline 12 & 6.97 & 0 & 6.92 & 1 & 8.88 & 2 & 0.197 & 0.336 & 0.702 & 1.645 & -1.649 & 2.751 \\
\hline 13 & 0 & 0.28 & 7.45 & 1 & 2 & 2.45 & 0.326 & 0.31 & 0.153 & 1.154 & 2.46 & 2.774 \\
\hline 14 & 0 & 0.83 & 8 & 1 & 2 & 3.18 & 0.182 & 0.189 & 0.428 & 1.067 & 0.521 & -2.87 \\
\hline 15 & 0 & 1.35 & 1 & 1.28 & 2 & 3.85 & 0.432 & 0.34 & 0.419 & -3.125 & 1.095 & -1.94 \\
\hline 16 & 0 & 1.85 & 1 & 1.83 & 2 & 4.48 & 0.397 & 0.189 & 0.385 & -1.111 & -0.998 & -1.307 \\
\hline 17 & 0 & 2.38 & 1 & 2.35 & 2 & 4.87 & 0.385 & 0.41 & 0.464 & 0.976 & 3.127 & -1.007 \\
\hline 18 & 0 & 2.88 & 1 & 2.85 & 2 & 5.37 & 0.446 & 0.399 & 0.641 & 3.103 & 1.086 & -0.374 \\
\hline 19 & 0 & 3.42 & 1 & 3.38 & 2 & 5.97 & 0.441 & 0.384 & 0.645 & -0.997 & -1.003 & 0.353 \\
\hline 20 & 0 & 3.92 & 1 & 3.88 & 2 & 6.48 & 0.441 & 0.471 & 0.374 & 0.889 & -3.095 & 1.07 \\
\hline 21 & 0 & 4.45 & 1 & 4.42 & 2 & 6.85 & 0.446 & 0.42 & 0.401 & 3.068 & 1.063 & 1.29 \\
\hline 22 & 0 & 4.95 & 1 & 4.92 & 2 & 7.48 & 0.385 & 0.42 & 0.418 & -1.088 & -0.957 & 1.908 \\
\hline 23 & 0 & 5.48 & 1 & 5.45 & 2 & 8.15 & 0.397 & 0.471 & 0.447 & 0.993 & -3.075 & 2.725 \\
\hline 24 & 0 & 5.98 & 1 & 5.95 & 2 & 8.88 & 0.432 & 0.384 & 0.387 & 3.001 & 1.115 & -2.181 \\
\hline 25 & 0 & 6.5 & 1 & 6.48 & & & 0.182 & 0.399 & & -1.188 & -0.968 & \\
\hline 26 & 0 & 7.03 & 1 & 6.98 & & & 0.326 & 0.409 & & 1.023 & -3.003 & \\
\hline 27 & & & 1 & 7.5 & & & & 0.189 & & & 1.119 & \\
\hline 28 & & & 1 & 8.05 & & & & 0.34 & & & -0.96 & \\
\hline
\end{tabular}

rectangular or non-rectangular 2-D planar array lattices. For example, the formulation in [22] (equation (6)) shows an array thinning from 41 to 35 while attempting to match -17.5
Chebyshev patterns and 121 to 57 to achieve "flat-top region" patterns. The given approaches are valid for a planar array in contrast to the linear array in our presented method. Another 
TABLE II

EXAMPLE SUMMARY

\begin{tabular}{|c|c|c|c|}
\hline & Array 1 & Array 2 & Array 3 \\
\hline \multicolumn{4}{|c|}{ Computational aspects } \\
\hline$\overline{\text { CS Iterations along }[x, y]}$ & {$[15,18]$} & {$[20,18]$} & {$[19,21]$} \\
\hline CPU time (s) & 11.58 & 13.79 & 14.42 \\
\hline \multicolumn{4}{|c|}{ Power pattern results } \\
\hline$\overline{\text { Main beam (deg) }}$ & $-22.5^{\circ},-40^{\circ}$ & $35^{\circ}, 40^{\circ}$ & $10^{\circ},-10^{\circ}$ \\
\hline along $x$ & $-49.6,-49.7$ & $-62.0,-63.6$ & $-32.2,-31.9$ \\
\hline along $y$ & $-49.8,-54.9$ & $-50.2,-51.5$ & $-31.6,-31.8$ \\
\hline Deviation of along $x$ & $0^{\circ}, 1^{\circ}$ & $0^{\circ}, 0^{\circ}$ & $1^{\circ}, 1.5^{\circ}$ \\
\hline along $y$ & $0^{\circ}, 0^{\circ}$ & $0^{\circ}, 0^{\circ}$ & $0^{\circ}, 0.5^{\circ}$ \\
\hline
\end{tabular}

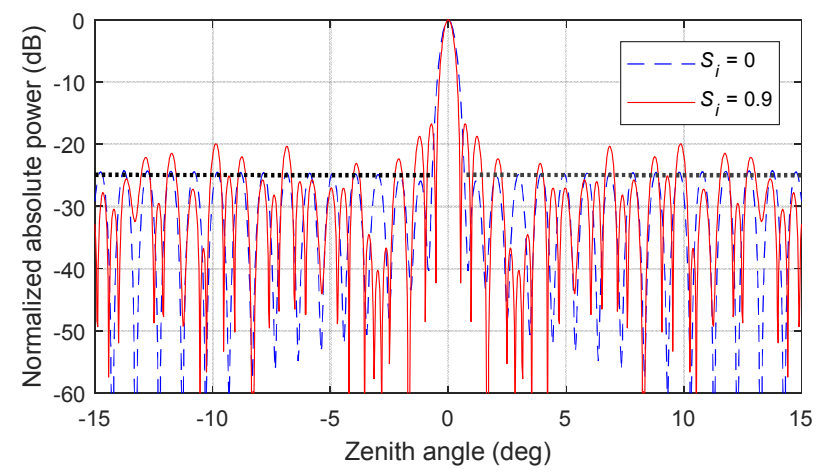

Fig. 7. 1-D principle plan cuts of resultant 2-D power patterns of a 136 element multiplicative antenna array.

recent effort by Bencivenni et al. [25] presented sub-dividing the circular aperture of an array by enforcing rotational symmetry using CS to achieve array thinning, again for a planar array lattice. Bencivenni's approach uses CS in 2-D with two degrees of freedom, and shows steering capability of $\pm 8^{\circ}$ for a Global Earth Coverage Application. In an attempt to draw an equitable comparison, SATCOM application specifications (Table I in [25]) are realized using multiplicative receiver array for a fixed beam case. By assuming isotropic antenna elements, we examined a 385 element array with 8-fold rotational symmetry. The proposed approach in this paper resulted in 136 elements array. The Out of Coverage angle of $0.79^{\circ}$ is realized by allocating first null at $\sim 0.8^{\circ}$ when $S_{i}=0$. Placement of null at the "Interbeam distance" of $1.06^{\circ}$ is further realized by the null allocation at $\sim 1^{\circ}$ when $S_{i}=0.9$. The results are shown in Fig. 7. The array topology was initially used to target a prescribed max. SLL $=-25 \mathrm{~dB}$, which first degraded to -24.5 $\mathrm{dB}$ after CS implementation, and then to $-16.7 \mathrm{~dB}$ at the cost of null placement at $\sim 1^{\circ}$. For the multi-fold circularly symmetric array, adequate sampling density is found to be $0.08 \lambda-0.03 \lambda$ [25], on the other hand, it has been noticed that the definitions in equation (10) and (11) in this paper does not limit the sampling density, provided that the computational complexity penalty is paid.

\section{CONCLUSION}

A novel approach for designing an antenna array of aggressively sparsely distributed antenna elements for multi beam recovery has been described. Multiplicative sparse array for single beam operation is discussed while the available space within the given array aperture is used to host multiple subarrays, projecting orthogonal fixed beams. The proposed approach is simple to implement, is computational efficient, and provides significant advantages in terms of system cost through reduction in the number of RF chains required. The method should find application in massive MIMO, beam modulation and electromagnetic imaging areas.

\section{ACKNOWLEDGEMENT}

Authors would like to thank Emmanuel Candes, Justin Romberg (Caltech), Stephen Boyd (Stanford) for public dissemination of their Convex Optimization material. Also thanks to Yuan Ding and Umair Naeem for discussion around directional modulation orthogonal vector synthesis and compressive sensing algorithm respectively. The work was sponsored by the UK Engineering and Physical Science Research Council EP/P000673/1, EP/N020391/1.

\section{REFERENCES}

[1] E. G. Larsson, O. Edfors, F. Tufvesson, and T. L. Marzetta, "Massive MIMO for next generation wireless systems," IEEE Commun. Mag., vol. 52, no. 2, pp. 186-195, 2014.

[2] A. L. Swindlehurst, E. Ayanoglu, P. Heydari, and F. Capolino, "Millimeter-wave massive MIMO: The next wireless revolution?," IEEE Commun. Mag., vol. 52, no. 9, pp. 56-62, 2014.

[3] Y. Ding and V. F. Fusco, "MIMO-inspired synthesis of directional modulation systems," IEEE Antennas Wirel. Propag. Lett., vol. 15, pp. 580-584, 2016.

[4] Y. Ding and V. Fusco, "A synthesis-free directional modulation transmitter using retrodirective array," IEEE J. Sel. Top. Signal Process., vol. 11, no. 2, pp. 428-441, 2017.

[5] Y. Ding and V. Fusco, "A review of directional modulation technology," Int. J. Microw. Wirel. Technol., vol. 8, no. 7, pp. 981-993, 2016.

[6] X. Zhuge and A. G. Yarovoy, "Study on two-dimensional sparse MIMO UWB arrays for high resolution near-field imaging," IEEE Trans. Antennas Propag., vol. 60, no. 9, pp. 4173-4182, 2012.

[7] M. Karaman, I. O. Wygant, Ö. Oralkan, and B. T. Khuri-Yakub, "Minimally redundant 2-D array designs for 3-D medical ultrasound imaging," IEEE Trans. Med. Imaging, vol. 28, no. 7, pp. 1051-1061, 2009.

[8] H. Wang and Y. Su, "Narrowband MIMO radar imaging with two orthogonal linear T/R arrays," in Signal Processing, 2008. ICSP 2008. 9th International Conference on, 2008, pp. 2513-2516.

[9] M. L. Skolnik, J. Sherman, and F. Ogg, "Statistically designed densitytapered arrays," IEEE Trans. Antennas Propag., vol. 12, no. 4, pp. 408$417,1964$.

[10] R. Redlich, "Iterative least-squares synthesis of nonuniformly spaced linear arrays," IEEE Trans. Antennas Propag., vol. 21, no. 1, pp. 106$108,1973$.

[11] R. Michelson and J. Schomer, "A radiation pattern synthesis technique for end-fire arrays," in IRE International Convention Record, New York, NY, USA, 1966, vol. 12, pp. 211-219.

[12] P. A. Matthews and K. A. Essuman, "Design of thinned arrays using the array-excitation autocorrelation function," in Proceedings of the Institution of Electrical Engineers, 1968, vol. 115, no. 2, pp. 253-255.

[13] Z. Lin, W. Jia, M. Yao, and L. Hao, "Synthesis of sparse linear arrays using vector mapping and simultaneous perturbation stochastic approximation," IEEE Antennas Wirel. Propag. Lett., vol. 11, pp. 220$223,2012$.

[14] J. Corcoles and M. A. Gonzalez, "Efficient combined array thinning and weighting for pattern synthesis with a nested optimization scheme," IEEE Trans. Antennas Propag., vol. 60, no. 11, pp. 5107-5117, 2012.

[15] X. Wang, E. Aboutanios, and M. G. Amin, "Thinned array beampattern synthesis by iterative soft-thresholding-based optimization algorithms," IEEE Trans. Antennas Propag., vol. 62, no. 12, pp. 6102-6113, 2014.

[16] W. P. Du Plessis and A. Bin Ghannam, "Improved seeding schemes for interleaved thinned array synthesis," IEEE Trans. Antennas Propag., vol. 62, no. 11, pp. 5906-5910, 2014.

[17] J. S. Petko and D. H. Werner, "Pareto optimization of thinned planar arrays with elliptical mainbeams and low sidelobe levels," IEEE Trans. Antennas Propag., vol. 59, no. 5, pp. 1748-1751, 2011. 
[18] X.-K. Wang, Y.-C. Jiao, and Y.-Y. Tan, "Synthesis of large thinned planar arrays using a modified iterative Fourier technique," IEEE Trans. Antennas Propag., vol. 62, no. 4, pp. 1564-1571, 2014.

[19] C.-H. Jang, F. Hu, F. He, J. Li, and D. Zhu, "Low-Redundancy Large Linear Arrays Synthesis for Aperture Synthesis Radiometers Using Particle Swarm Optimization," IEEE Trans. Antennas Propag., vol. 64, no. 6, pp. 2179-2188, 2016.

[20] K. Rezer, C. Klickow, and A. F. Jacob, "Thinned MIMO frame-arrays for radar imaging," in Microwave Symposium Digest (MTT), 2012 IEEE MTT-S International, 2012, pp. 1-3.

[21] K. Rezer, W. Gropengießer, and A. F. Jacob, "Particle swarm optimization of minimum-redundancy MIMO arrays," in Microwave Conference (GeMIC), 2011 German, 2011, pp. 1-4.

[22] M. D'Urso, G. Prisco, and R. M. Tumolo, "Maximally sparse, steerable, and nonsuperdirective array antennas via convex optimizations," IEEE Trans. Antennas Propag., vol. 64, no. 9, pp. 3840-3849, 2016.

[23] Y. Liu, Z. Nie, and Q. H. Liu, "Reducing the number of elements in a linear antenna array by the matrix pencil method," IEEE Trans. Antennas Propag., vol. 56, no. 9, pp. 2955-2962, 2008.

[24] M. Miller, J. Hinze, M. Saquib, and A. J. Blanchard, "Adjustable transmitter spacing for MIMO radar imaging with Compressed Sensing," IEEE Sens. J., vol. 15, no. 11, pp. 6671-6677, 2015.

[25] C. Bencivenni, M. V Ivashina, R. Maaskant, and J. Wettergren, "Synthesis of maximally sparse arrays using compressive sensing and full-wave analysis for global earth coverage applications," IEEE Trans. Antennas Propag., vol. 64, no. 11, pp. 4872-4877, 2016.

[26] S. Karimkashi and A. A. Kishk, "Invasive weed optimization and its features in electromagnetics," IEEE Trans. Antennas Propag., vol. 58, no. 4, pp. 1269-1278, 2010.

[27] O. Quevedo-Teruel and E. Rajo-Iglesias, "Ant colony optimization in thinned array synthesis with minimum sidelobe level," IEEE Antennas Wirel. Propag. Lett., vol. 5, no. 1, pp. 349-352, 2006.

[28] C. Cui, W. T. Li, X. T. Ye, and X. W. Shi, "Hybrid Genetic Algorithm and Modified Iterative Fourier Transform Algorithm for Large Thinned Array Synthesis," IEEE Antennas Wirel. Propag. Lett., 2017.

[29] J. Rubio, J. Córcoles, J. F. Izquierdo, and R. Gómez-Alcalá, “Array Thinning of Coupled Antennas Based on the Orthogonal Matching Pursuit Method and a Spherical-Wave Expansion for Far-Field Synthesis," IEEE Trans. Antennas Propag., vol. 63, no. 12, pp. 5425$5432,2015$.

[30] G. Oliveri, E. T. Bekele, F. Robol, and A. Massa, "Sparsening conformal arrays through a versatile \$ BCS \$-based method," IEEE Trans. Antennas Propag., vol. 62, no. 4, pp. 1681-1689, 2014.

[31] B. Y. Mills and A. G. Little, "A high-resolution aerial system of a new type," Aust. J. Phys., vol. 6, no. 3, pp. 272-278, 1953.

[32] R. Vescovo, "Consistency of constraints on nulls and on dynamic range ratio in pattern synthesis for antenna arrays," IEEE Trans. Antennas Propag., vol. 55, no. 10, pp. 2662-2670, 2007.

[33] Y. Ding and V. Fusco, "Orthogonal vector approach for synthesis of multi-beam directional modulation transmitters," IEEE Antennas Wirel. Propag. Lett., vol. 14, pp. 1330-1333, 2015.

[34] W. P. M. N. Keizer, "Synthesis of thinned planar circular and square arrays using density tapering," IEEE Trans. Antennas Propag., vol. 62, no. 4, pp. 1555-1563, 2014.

[35] G. Prisco and M. D'Urso, "Maximally sparse arrays via sequential convex optimizations," IEEE Antennas Wirel. Propag. Lett., vol. 11, pp. 192-195, 2012.

[36] D. Brandwood, Fourier transforms in radar and signal processing. Artech House, 2012.

[37] R. A. Horn and C. R. Johnson, Matrix Analysis. 2012.

[38] H. M. Aumann, "A pattern synthesis technique for multiplicative arrays," PIERS Proceedings, Cambridge, USA, pp. 864-867, 2010.

[39] S. S. Chen, D. L. Donoho, and M. A. Saunders, "Atomic decomposition by basis pursuit," SIAM Rev., vol. 43, no. 1, pp. 129-159, 2001.

[40] E. Van Den Berg and M. P. Friedlander, "Probing the Pareto frontier for basis pursuit solutions," SIAM J. Sci. Comput., vol. 31, no. 2, pp. 890912, 2008.

[41] E. Candes and J. Romberg, "11-magic: Recovery of sparse signals via convex programming," URL www. acm. caltech. edu/l1magic/downloads/l1magic. pdf, vol. 4, p. 14, 2005.

[42] F. Ares, A. Vieiro, E. Moreno, and S. R. Rengarajan, "Optimization of aperture distributions for difference patterns," J. Electromagn. waves Appl., vol. 10, no. 3, pp. 383-402, 1996.

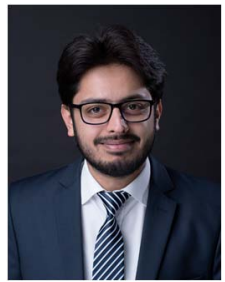

Muhammad Ali Babar Abbasi (S'14-M'17) received the B.S. degree from the CIIT, Pakistan, in 2011, the M.S. degree from the National University of Sciences and Technology (NUST), Islamabad, in 2013, and Ph. D. from Frederick University, Nicosia, Cyprus (European) in 2017, all in electrical engineering. He is currently a Research Fellow with the Centre of Wireless Innovation (CWI) at Queen's University Belfast (QUB), Belfast, U.K.

He has authored and co-authored more than 25 journal and conference papers. He was a recipient of the Erasmus Mundus INTACT Doctoral Scholarship by the European Union in 2014 and COST VISTA STSM grand in 2016. He was also the finalist in the Ericsson Innovation Awards in 2016 and was enlisted as IET young professional in 2017.

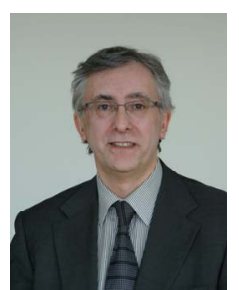

Vincent Fusco (S'82-M82-SM'96-F'04) received the Bachelor's (Hons.) degree in electrical and electronic engineering, the Ph.D. degree in microwave electronics, and the D.Sc. degree all from Queen's University Belfast (QUB), Belfast, U.K., in 1979, 1982, and 2000, respectively.

His work was focused on advanced front-end architectures with enhanced functionality. $\mathrm{He}$ is currently Chief Technology Officer of the Institute of Electronics, Communications and Information Technology, QUB. He has authored more than 450 scientific papers in major journals and in referred international conferences. He has authored two textbooks, holds patents related to self-tracking antennas, and has contributed invited papers and book chapters. His current research interests include active antenna and front-end MMIC techniques. Professor Fusco is a Fellow of the Institute of Electrical and Electronics Engineers, the Institution of Engineering and Technology, the Royal Academy of Engineers, and the Royal Irish Academy. In 2012, he received the IET Senior Achievement Award and the Mountbatten Medal. He serves on the Technical Program Committee of various international conferences, including the European Microwave Conference.

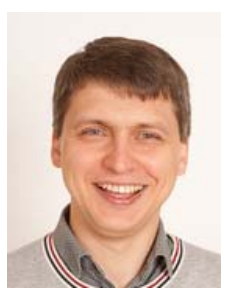

Dmitry Zelenchuk (S'02-M'05) received the Ph.D. degree in radiophysics from Rostov State University, Rostov, Russia, in 2004.

From 2003 to 2005, he was a Lecturer with the Department of Applied Electrodynamics and Computer Modelling, Rostov State University, Russia. Currently, he is a Lecturer with the Centre for Wireless Innovations, ECIT, Queen's University Belfast, U.K. His research interests include electromagnetic field theory, material characterization, millimeter-wave circuits, antennas, and advanced packaging, propagation in complex environments, and various physical phenomena of plasmonic and nanostructures. He has authored and coauthored more than 80 journal and conference papers and a book chapter and been a session chair at scientific conferences. 